\title{
Efecto de la arginina 8\%-carbonato de calcio y del fluoruro de sodio al $5 \%$ en la reducción de la hipersensibilidad dentinaria post terapia periodontal: ensayo clínico
}

\author{
Effect of $8 \%$ arginine, calcium carbonate and $5 \%$ sodium fluoride on the reduction of \\ the dentine hipersensitivity post periodontal therapy: clinical trial
}

Márquez $\mathrm{M}^{1}$, Quintero $\mathrm{A}^{2}$, Sanz A², Ramírez V³, Inostroza $\mathrm{C}^{4}$, Chaparro $\mathrm{A}^{2}$

\begin{abstract}
RESUMEN
Objetivo: El objetivo de este estudio clínico fue evaluar y comparar la eficacia en la reducción de la hipersensibilidad dentinaria de la arginina al 8\%carbonato de calcio, monofluorfosfato (1.450 ppm) en comparación con un grupo control tratado en base a un barniz de flúor (22.600 ppm) y un dentífrico fluorado $(1.450 \mathrm{ppm}), 3$ veces al día durante un minuto, en pacientes con hipersensibilidad radicular en forma inmediata a la terapia periodontal mecánica (medición basal) y a las 4 semanas respectivamente. Materiales y Método: Estudio clínico aleatorizado de grupos paralelos, con 4 semanas de seguimiento, en el cual se reclutaron 20 pacientes con diagnóstico de periodontitis crónica leve a moderada, en tratamiento periodontal mecánico con presencia de recesiones gingivales a nivel de incisivos, caninos o premolares. A todos ellos, previa firma de consentimiento informado y en forma inmediata a los pulidos radiculares, se les aplicó el test térmico de chorro de aire con la jeringa de aire de la unidad dental a nivel de las recesiones gingivales con hipersensibilidad. La molestia o dolor fue determinado mediante la utilización de una escala visual análoga, realizada en forma inmediata a los pulidos radiculares (medición basal). Los pacientes fueron aleatoriamente asignados a uno de los siguientes grupos: grupo control, se les aplicó barniz de flúor (22.600 ppm) en todas las recesiones gingivales y se les indicó un dentífrico fluorado (1.450 ppm); grupo experimental, a quienes se les aplicó pasta tópica de arginina y carbonato de calcio (8\%) a nivel de las recesiones gingivales, complementado con el uso de un dentífrico cuyo componente activo es la arginina al 8\%-carbonato de calcio. Todos los pacientes fueron evaluados 4 semanas posterior a la aplicación y utilización domestica de los agentes des-sensibilizantes por un único examinador. Resultados: Ambos grupos experimentaron una disminución en la sensibilidad dentinaria, sin embargo, se logró una mayor reducción del dolor en aquellos pacientes que utilizaron la terapia de arginina al $8 \%$ y carbonato de calcio, con un $60 \%$ de los individuos sin dolor y con una mediana final de 0 en comparación al grupo con barniz de flúor al $5 \%$ y un dentrífico fluorado que mantuvieron en el $100 \%$ de los sujetos algún grado de dolor y presentando una mediana final en la escala VAS de 2.
\end{abstract}

Rev. Clin. Periodoncia Implantol. Rehabil. Oral Vol. 4(1); 22-25, 2011.

Palabras clave: Terapia periodontal, recesión gingival, hipersensibilidad dentinaria, arginina, carbonato, fluoruro de sodio.

\section{ABSTRACT}

Aim: The aim of this clinical study was to evaluate and to compare the efficacy in reducing the dentine hypersensitivity of an $8 \%$ arginine, calcium carbonate, $1.450 \mathrm{ppm}$ sodium monofluorophosphate dentifrice with topical application and twice-daily brushing, with a negative control toothpaste containing calcium carbonate and $1.450 \mathrm{ppm}$ fluoride as MFP, after periodontal treatment (baseline) and four weeks respectively. Methods: A fourweek clinical study with 20 subjects with diagnosis of slight to moderate chronic periodontitis under mechanical periodontal treatment and presence of gingival recessions in incisors, canines or premolars, stratified based on baseline tooth sensitivity. Subjects brushed with either a 1.450 ppm fluoride dentifrice or a marketed $8 \%$ arginine calcium carbonate dentifrice twice daily for approximately one minute. At screening, baseline and weeks four, subjects' tooth sensitivity was determined through both evaporative (Schiff and Visual Analogue Scale [VAS]). The same examiner throughout the study performed subject assessments using each stimulus. Results: Both subject groups exhibited significant reductions from baseline to four weeks in dentine hypersensitivity. However, patients who received $8 \%$ arginine calcium carbonate therapy, exhibited a greater reduction, with a $60 \%$ of individuals lacking of pain and with a final median of 0 in comparison with the $5 \%$ fluoride varnish and fluoride toothpaste group in which a $100 \%$ of subjects felt some degree of pain, presenting a final medium VAS scale of 2.

Rev. Clin. Periodoncia Implantol. Rehabil. Oral Vol. 4(1); 22-25, 2011.

Key words: Periodontal therapy, gingival recession, dental hypersensitivity, arginine, carbonate, sodium fluoride.

\section{INTRODUCCIÓN}

La hipersensibilidad dentinaria se define como un dolor corto, agudo, punzante y localizado(1), como respuesta a estímulos químicos, térmicos, táctiles o mecánicos sobre la dentina expuesta. Su etiología es múltiple, sin embargo, se asocia con la pérdida de esmalte (producto de abrasión, atrición, caries, etc.) o debido a la presencia de recesiones gingivales (enfermedad periodontal, cepillado traumático, etc.) $)^{(2)}$.

La prevalencia de la hipersensibilidad varía entre un 9\%-55\% de la población según distintas investigaciones ${ }^{(1,3,4,5)}$. El rango etáreo en el cual aumenta los cuadros de hipersensibilidad se ubica entre la tercera y cuarta década de vida, siendo más prevalente en mujeres que en hombres $^{(6,7)}$. En cuanto a su distribución en la arcada dentaria, afectaría principalmente las superficies vestibulares de caninos y premolares

1. Cirujano Dentista. Universidad de los Andes. Chile.

2. Cirujano Dentista. Especialista en Periodoncia. Área de Periodoncia. Docente Universidad de los Andes. Chile.

3. Cirujano Dentista. Magíster Salud Pública. Área de Epidemiología. Docente Universidad de los Andes. Chile.

4. Bioquímica. Magíster en Microbiología. Centro de Investigación de Biología y Regeneración Oral (CIBRO). Universidad de los Andes. Chile.

Correspondencia autor: Alejandra Chaparro Padilla. achaparro@uandes.cl / chaparro.ale@gmail.com. Universidad de los Andes. Chile. Trabajo recibido el 28/02/2011. Aprobado para su publicación el 27/03/2011. 
superiores, seguido por incisivos y molares mandibulares ${ }^{(1,2,3)}$.

Existen diversas hipótesis que explican los mecanismos biológicos por los cuales se produciría la hipersensibilidad, sin embargo, uno de los mecanismos propuestos más aceptados en la actualidad, es la teoría hidrodinámica del dolor ${ }^{(4)}$. Ésta consiste en que los fluidos existentes al interior de los túbulos dentinarios se encontrarían en equilibrio osmótico, conectados en forma directa con la pulpa dentaria. La presencia de estímulos externos mecánicos, químicos, térmicos o táctiles, alterarían el equilibrio osmótico, afectando los baro-receptores, generando un impulso nervioso por parte de las fibras nerviosas que se encuentran en la pulpa de la pieza dentaria ${ }^{(1,8)}$

Tanto la pérdida de inserción de los tejidos periodontales en pacientes diagnosticados con periodontitis como su respectiva terapia mecánica (remoción mecánica del biofilm supragingival y subgingival) representan factores asociados a la presencia de hipersensibilidad dentinaria. Investigaciones previas reportan entre un $9 \%-23 \%$ de hipersensibilidad cervical en forma previa a la terapia convencional, situación que aumenta entre un $54 \%$ a $55 \%$ posterior a la terapia periodontal. Este dolor incrementa entre la primera y tercera semana, para luego ir disminuyendo hasta la octava semana, en donde se mantendría estable, como una molestia de tipo crónico ${ }^{(1,3,5)}$. Adicionalmente, en forma posterior a la terapia mecánica de destartraje subgingival y pulido radicular, se desinflaman los tejidos periodontales y producto de la pérdida de los tejidos de soporte periodontal, los tejidos gingivales se retraen, presentándose ciertas secuelas como la presencia de recesiones del tejido gingival, con la consecuente exposición radicular, la cual, producto de la instrumentación radicular, se encuentra desprovista de cemento, exponiendo los túbulos dentinarios al medio oral, gatillando la sensibilidad radicular, frente a estímulos térmicos, químicos y mecánicos ${ }^{(5)}$.

Recientemente, se ha propuesto un nuevo agente para el tratamiento de la hipersensibilidad dentinaria, en base a los agentes terapéuticos: arginina al $8 \%$ y a carbonato de calcio. Según sus fabricantes, la aplicación tópica de este producto en las áreas hipersensibles disminuiría significativamente los niveles de dolor y sensibilidad cervical luego de una sola aplicación ${ }^{(10)}$.

La arginina es un aminoácido, con carga positiva, a un $\mathrm{pH}$ fisiológico de 6.5 a 7.5 y se obtiene de los alimentos o a través del ciclo de la urea, poseería un efecto benéfico participando en la oclusión de los túbulos dentinarios abiertos, resistente a la exposición de ácidos provenientes de la dieta, con propiedades de baja conductancia hidráulica (un $63 \%$ menor luego de su aplicación) y con una composición química baja en nitrógeno y carbono, pero alta de calcio, oxígeno y fósforo ${ }^{(10)}$.

El carbonato de calcio ( $\mathrm{CaCO} 3)$ es un compuesto químico que se encuentra de forma abundante en la naturaleza. Es poco soluble, y en medios acuosos como el agua, puede disociarse en carbonato y calcio(11). $^{(11)}$

La arginina, el bicarbonato y el carbonato de calcio, interaccionan generando una obstrucción física/mecánica del túbulo dentinario expuesto, logrando el alivio del paciente con hipersensibilidad dentinaria. Al cepillarse, la arginina contenida en el dentífrico, en conjunto con la saliva del paciente, reaccionarían con el bicarbonato y el carbonato de calcio, formando una estructura central de bicarbonato de arginina el cual es altamente soluble, rodeado de carbonato de calcio el cual es poco soluble. Esta estructura tendría propiedades adhesivas por lo que formaría una mezcla que no solo obstruiría los túbulos dentinarios abiertos, sino que también se adheriría a las paredes de los tubulillos, propiedad otorgada debido a la alcalinidad de la molécula(10,11).

El objetivo del presente estudio clínico fue evaluar el efecto de la arginina al 8\%-carbonato de calcio, en la reducción de la hipersensibilidad dentinaria en comparación a la aplicación de fluoruros a un grupo de pacientes con sensibilidad dentinaria como secuela del tratamiento periodontal, el cual fue evaluado 4 semanas posterior a su aplicación.

\section{MATERIALES Y MÉTODO}

Se desarrolló un ensayo clínico, aleatorizado controlado con 2 grupos paralelos (1:1).

\section{Selección de Muestra}

Fueron seleccionados 20 sujetos con diagnóstico de periodontitis crónica, que acudieron a la Clínica de Periodoncia del Centro de Salud de la Universidad de los Andes, todos los pacientes ingresaron voluntariamente al estudio y firmaron previamente un consentimiento informado. El estudio contó con la aprobación del comité de ética de la Facultad de Odontología de la Universidad de los Andes.

Todos los pacientes recibieron tratamiento periodontal convencional no quirúrgico consistente en: instrucción en técnicas de higiene oral, destartraje supragingival, subgingival y pulido radicular, además, debieron cumplir con todos los criterios de inclusión y exclusión establecidos. Los criterios de inclusión fueron: voluntarios entre 18 y 70 años, con buena salud que no contraindiquen su participación en el estudio; diagnóstico con periodontitis crónica generalizada leve o moderada; índice de placa bacteriana de Löe y Sillness menor al $20 \%$; con un mínimo de 2 piezas dentarias anteriores (incisivos, caninos o premolares) con hipersensibilidad, y concomitante recesión gingival, erosión o anfracción en la superficie cervical vestibular radicular en forma posterior a la terapia periodontal; con resultado de la escala de VAS entre 1-10 con dolor localizado en zona cervical de piezas dentarias, luego de la aplicación del chorro de aire; y dispuestos al seguimiento durante la realización del estudio. Los criterios de exclusión fueron: utilización de alguno de los protocolos de terapia des-sensibilizantes actualmente reconocidos como tales; uso crónico de medicamentos (AINE u analgésicos, anticonvulsivantes, antihistamínicos, antidepresivos o sedantes); embarazo o lactancia; con enfermedades mentales; con alergia a los productos a utilizar: Flúor Duraphat, dentífrico Colgate ${ }^{\circledR}$ Sensitive Pro-Alivio ${ }^{\mathrm{TM}}$, Colgate Total ${ }^{\circledR} 12$ Professional Clean; con desórdenes alimenticios como bulimia, anorexia; con excesiva ingesta de alimentos ácidos; con auto-reporte de reflujo gastroesofágico; con cirugía periodontal en los 3 meses anteriores al estudio; con dientes con patologías como pulpitis reversible, pulpitis irreversible, necrosis pulpar y/o abscesos dentoalveolares; con dientes soporte de prótesis removible o coronadas; con restauraciones recientes, extensas o defectuosas; con caries, fracturas, movilidad superior a grado 1; con fracturas de esmalte; con aparatos ortodóncicos o con dientes con restauraciones clase $\mathrm{V}$ en zona cervical. A todos los pacientes se les entregó un cepillo de filamentos suaves y una pasta dental, estos fueron donados por la empresa Colgate-Palmolive ${ }^{\circledR}$

En forma inmediatamente posterior a la terapia periodontal se seleccionaron aquellos voluntarios que presentaron hipersensibilidad cervical producto de la terapia periodontal. Luego, los sujetos fueron distribuidos aleatoriamente a los grupos test y control, quedando conformados por 10 sujetos cada uno de los grupos (Figura 1). La aleatorización se realizó a través del programa computacional STATA 10.0 de manera centralizada, manteniéndose oculta la secuencia de aleatorización

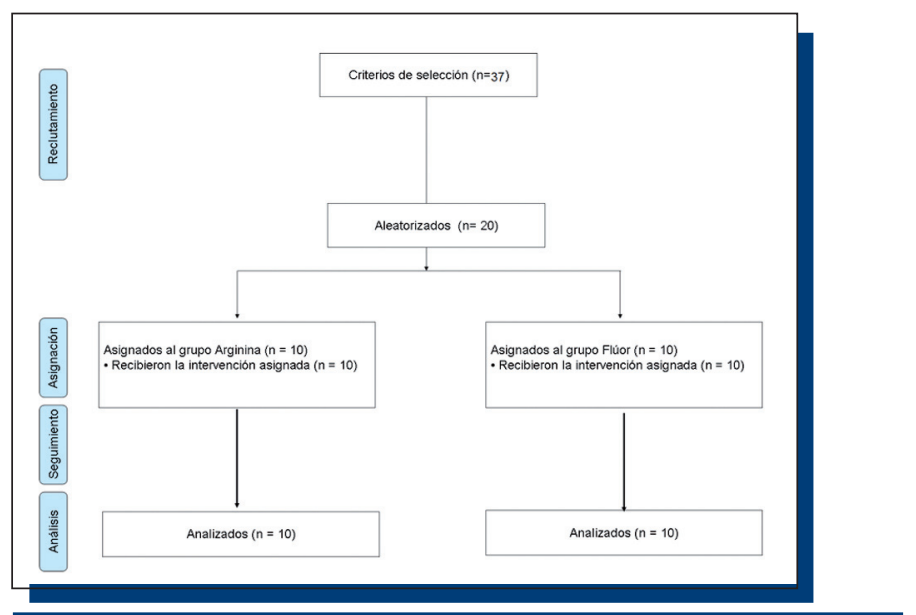

Figura 1. Diagrama de flujo del estudio.

\section{Determinación de los Niveles de Sensibilidad}

Todos los pacientes fueron evaluados por un solo examinador calibrado a todas las piezas dentarias indicadas por los sujetos participantes con presencia de hipersensibilidad dentinaria, para ello se aplicó el test evaporativo, este consiste en la aplicación de aire, realizado con jeringa triple estándar de sillón dental Sirona ${ }^{\circledR}$ de 60 psi (5+- psi) y $70^{\circ} \mathrm{F}\left(+-3^{\circ} \mathrm{F}\right)$, por 3 segundos, a $1 \mathrm{~cm}$ de la superficie cervical vestibular 
radicular y perpendicular a la pieza dentaria y con aislación previa de las piezas dentarias vecinas con tórulas de algodón.

\section{Escala de VAS}

La cuantificación del dolor se realizó a través de la Escala Visual Análoga (VAS), utilizando un código numérico del 0 al 10: en la cual; $0=$ no hay dolor; 1 a 10 , siendo 1 molestia leve, aumentando gradualmente hasta 10 con un incomodidad importante por más de 10 segundos (Figura 2).

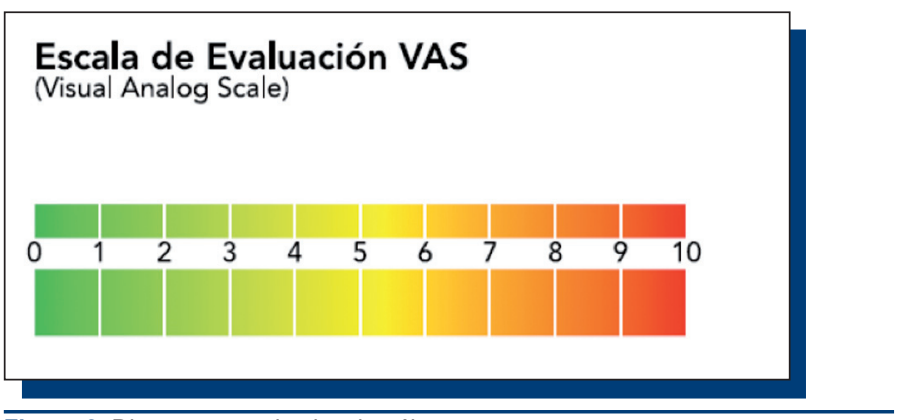

Figura 2. Diagrama escala visual análoga.

\section{Aplicación de los Agentes Terapéuticos}

Un alumno entrenado, distinto del examinador, aplicó los agentes en estudio sobre las piezas afectadas, de modo que los grupos fueron tratados de la siguiente manera:

- Grupo Test: Se utilizó pasta de aplicación tópica Colgate® Sensitive ProAlivio $^{\mathrm{TM}}$ sobre la superficie radicular expuesta al medio oral de las piezas dentarias con sensibilidad con una copa de goma o escobilla, durante 30 segundos por diente, al terminar el pulido radicular. Se entregó al paciente pasta Colgate ${ }^{\circledR}$ Sensitive Pro-Alivio ${ }^{\mathrm{TM}}$ de uso doméstico o en casa, con indicación de cepillado de 1 minuto de duración, 3 veces al día con técnica de Charters.

- Grupo Control: Aplicación de barniz de fluoruro de sodio al $5 \%$, Duraphat $₫(22.600 \mathrm{ppm})$, por parte del alumno tratante, en las superficies radiculares expuestas de las piezas sensibles. Se entregó al paciente pasta con fluoruro de sodio en una concentración de 1.450 ppm de F, de aplicación casera, indicación de cepillado de 1 minuto de duración, 3 veces al día con técnica de Charters.

Para la aplicación de ambos agentes se siguió el protocolo descrito a continuación en la zona cervical con exposición radicular:

1. Limpieza previa de las piezas dentarias con exposición radicular con escobilla, agua y seda dental.

2. Secado y aislación relativa de las piezas dentarias, con algodón y eyector.

3. Aplicación de una capa fina de barniz de flúor Duraphat $\circledast$ en el grupo control, con pincel, según instrucciones del fabricante.

4. Aplicación de pasta Colgate ${ }^{\circledR}$ Sensitive Pro-Alivio ${ }^{\mathrm{TM}}$ de aplicación clínica, con copas de goma, según instrucciones del fabricante.

5. Información al paciente, por escrito, de las indicaciones post aplicación: no enjuagarse, no ingerir líquidos ni comer nada por tres horas, no cepillarse por 12 horas ni utilizar seda dental por 24 horas.

\section{Análisis Estadístico}

El análisis estadístico comenzó con una descripción de las variables evaluadas. Aquellas variables nominales fueron descritas mediante tablas con frecuencias absolutas y relativas. La sensibilidad medida como variable ordinal, se describió a través de la mediana, el rango intercuartílico, el valor mínimo y máximo, y se compararon según grupo de tratamiento al inicio y al final de él a través de la prueba de Mann Whitney. La comparación de esta misma variable en cada grupo antes y después del tratamiento se evaluó con la prueba de Wilcoxon para muestras pareadas. Un $p<0.05$ fue considerado estadísticamente significativo y todos los valores $p$ fueron bilaterales. Se evaluó también la variable sensibilidad como dicotómica, reportando el riesgo relativo (RR) y diferencia de riesgo, con sus respectivos intervalos de confianza
(IC) del 95\%. Por último, se calculó la potencia del estudio a dos colas con un nivel de significación de 0.05 con la diferencia de proporciones obtenidas. El análisis estadístico se realizó con STATA 10.0

\section{RESULTADOS}

El rango de edad de los pacientes incluidos en el estudio, fue entre 20 y 53 años. Un $80 \%$ de los sujetos, correspondió al género femenino y un $20 \%$ al género masculino. Tanto en el grupo que utilizó el sistema el protocolo con arginina como el grupo control no se observaron diferencias entre los valores de la escala de VAS entre los grupos control y test en la evaluación inicial (Tabla 1).

Tabla 1. Descripción inicial de los pacientes según sexo y nivel de sensibilidad.

\begin{tabular}{|c|c|c|}
\hline Variable & Proalivio (N=10) & Control (N=10) \\
\hline $\begin{array}{c}\text { Sexo } \\
\text { Femenino }\end{array}$ & $8(80 \%)$ & $8(80 \%)$ \\
\hline Masculino & $2(20 \%)$ & $2(20 \%)$ \\
\hline $\begin{array}{c}\text { Sensibilidad al inicio } \\
\text { Sensibilidad (VAS) }\end{array}$ & $5(2-7)$ & $5(4-7)$ \\
\hline
\end{tabular}

* La información corresponde a la mediana (mínimo - máximo) o frecuencias (\%).

En la segunda medición, realizada a las 4 semanas, se observó que los valores de sensibilidad disminuyeron en ambos grupos, con un valor $p=0.0048$ para el grupo que utilizó el agente arginina/carbonato de calcio y un valor $p=0.0072$ en el grupo control (Prueba Wilcoxon pareada).

Todos los sujetos pertenecientes al grupo control presentaron sensibilidad residual a las 4 semanas post terapia a diferencia del grupo tratado con arginina/carbonato de calcio, en donde un $60 \%$ de pacientes no presentó sensibilidad dentinaria (Tabla 2).

Tabla 2. Descripción inicial de la sensibilidad como variable dicotómica y ordina antes y después de los tratamientos.

\begin{tabular}{|c|c|c|}
\hline Variable & Proalivio (N=10) & Control (N=10) \\
\hline $\begin{array}{c}\text { Sensibilidad (VAS) } \\
\text { Inicio }\end{array}$ & $5(2-7)$ & $5(4-7)$ \\
\hline $\begin{array}{c}\text { A las 4 semanas } \\
\text { Sensibilidad a las 4 semanas } \\
\text { No (VAS=0) }\end{array}$ & $0(0-5)$ & $2(2-5)$ \\
\hline Sí (VAS>=1) & $6(60 \%)$ & $0(0 \%)$ \\
\hline
\end{tabular}

* La información corresponde a la mediana (mínimo - máximo) o frecuencias (\%)

La sensibilidad final medida en escala ordinal, fue mayor en el grupo control que en el con tratamiento con arginina (Prueba Mann Whitney, $p=0.0085$ ), como se observa en la Figura 3. El tratamiento con arginina comparado con el de flúor presentó un riesgo relativo de 0,4 (IC $95 \%=0.19-0.85$ ), es decir el riesgo que tiene un individuo en el grupo con arginina de presentar dolor (tener un valor distinto de cero) es $40 \%$ del riesgo que tienen los del grupo control. Asimismo, se encontró una diferencia del riesgo de tener dolor de -0.6 (IC 95\% = -0.90 - -0.30). La potencia del estudio para esta diferencia fue de $74 \%$. 


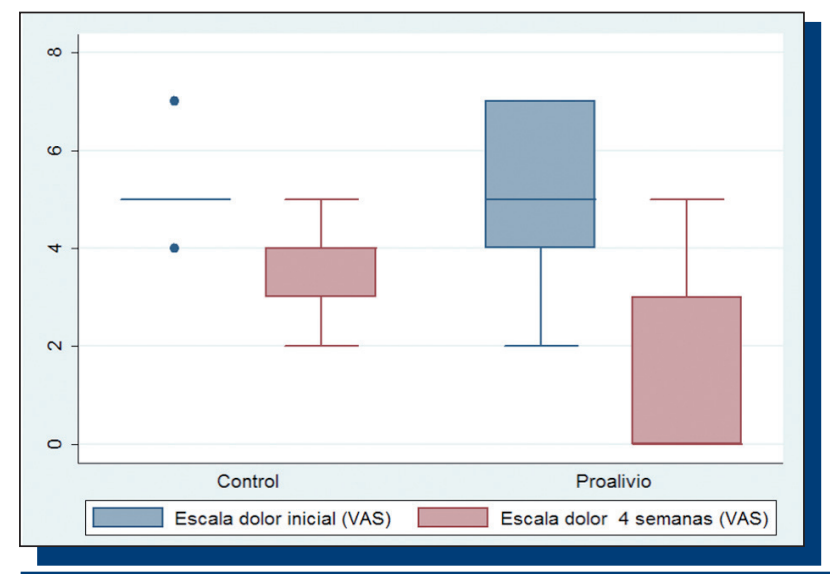

Figura 3. Comparación de la sensibilidad dentinaria entre los grupos test y control, antes y después del tratamiento.

\section{DISCUSIÓN}

En el presente estudio podemos observar que existió una disminución de la sensibilidad dentinaria medido a las 4 semanas post terapia periodontal y de aplicación de los agentes terapéuticos para el manejo de la sensibilidad. Ambos grupos experimentaron una mejoría en su condición de hipersensibilidad, estos resultados, concuerdan con publicaciones previas, las cuales nos indican que un porcentaje de la sensibilidad cervical disminuye sin realizar intervenciones terapéuticas entre la cuarta y octava semana post terapia periodontal ${ }^{(1,3,5)}$. Aunque ambos grupos disminuyeron los niveles de sensibilidad inicialmente registrados, se mostró una mayor eficacia en la reducción del dolor en la terapia que utilizó arginina al 8\% y carbonato de calcio en comparación con la aplicación barniz de fluoruro de sodio. Sin embargo, a pesar de que los resultados son positivos, se necesitaría un mayor tamaño de muestra para obtener una mayor potencia y mayor precisión de sus resultados.

Estudios recientes demuestran una reducción de la sensibilidad dentinaria similar a los encontrados en nuestra investigación, la cual comienza a disminuir luego de la aplicación del agente con arginina/ carbonato de calcio, con una duración aproximada de 28 días 12.

El flúor también ha demostrado ser capaz de lograr una disminución de la sensibilidad dentinaria obstruyendo los túbulos dentinarios con el depósito de flúor en ellos, sin embargo, es necesario un tiempo mayor para que esto se logre, en comparación a este nuevo producto que logra niveles de reducción de sensibilidad incluso luego de una y única aplicación ${ }^{(8,13,14)}$

El presente estudio apoya la evidencia científica en relación al uso de la arginina y el carbonato de calcio para el tratamiento de la hipersensibilidad dentinaria, obteniéndose valores de reducción que son estadísticamente significativos, logrando incluso la reducción completa de la sensibilidad en el grupo tratado con arginina/carbonato de calcio a diferencia del grupo tratado con fluoruros, en el cual a pesar de que existió una reducción en los valores de la escala VAS para la hipersensibilidad, estos se mantuvieron en un alto porcentaje de los pacientes, incluso 1 mes posterior a la terapia periodontal.

Dentro de las limitaciones del estudio se encuentra la medición en la escala de VAS, ya que la ejecuta el paciente y éste no estaba enmascarado al tratamiento, es decir, el paciente conocía el tipo de terapia a la que estaba expuesto. Por otra parte, no es posible tener certeza del cumplimiento de las indicaciones, sin embargo esto puede haber ocurrido por azar tanto en el grupo control como en el grupo test. Al entregar la pasta al sujeto puede haber existido sesgo por una información por parte del paciente del contenido de la pasta, ya sea por la publicidad o por el acceso a internet, sugestionando al paciente.

El presente estudio no estimó un cálculo de tamaño de muestra previo dado que no existía información de estudios previos que realizaran esta comparación, por lo que se desconocía las diferencias que se encontrarían entre ambos grupos. Sin embargo, los resultados entregan información valiosa para poder realizar un estudio confirmatorio con un cálculo de tamaño de muestra apropiado.

Se sugiere en investigaciones posteriores utilizar encuestas para evaluar otras variables como: adherencia y cumplimiento del tratamiento; extender el período de seguimiento y generar un enmascaramiento con el objetivo que los pacientes desconozcan el tipo de tratamiento utilizado, lo que se podría lograr generando pastas idénticas en su presentación.

Este ensayo clínico indica que existe una mayor reducción de la sensibilidad por parte del dentrífico con arginina/carbonato de calcio al $8 \%$ luego de cuatro semanas de uso, llegando incluso a reducirla de forma total, lo que de confirmarse, implicaría la indicación de la arginina como agente coadyuvante en la terapia periodontal mecánica convencional con la finalidad de lograr una disminución de sensibilidad cervical, secuela producto tanto de la pérdida de los tejidos de soporte periodontal como también de la remoción del cemento contaminado durante la terapia periodontal mecánica.

\section{REFERENCIAS BIBLIOGRÁFICAS}

1. Von Troil B, Needleman I, Sanz M. A systematic review of the prevalence of root sensitivity following periodontal therapy. J Clin Periodontol, 2002; 29(3): 173-177.

2. West NX. Dentine Hipersensitivity: Preventive and therapeutic approaches to treatment. Periodontology 2000, 2008; 48: 31-41.

3. Chabanski MB, Gillam DG. Aetiology, prevalence and clinical features of cervical dentine sensitivity. J Oral Rehabil, 1997; 24(1): 15-19.

4. Brännström M. A hydrodynamic mechanism in the transmission of painproduced stimuli through the dentine. In: Anderson DJ editor. Sensory mechanisms in dentine. London: Pergamon Press. 1963: 73-79.

5. Bergenholtz G, Tamaro S, Wennström JL. Root-dentin sensitivity following non-surgical periodontal treatment. J Clin Periodontol, 2000; 27: 690-697.

6. Addy M, Embery G, Edgar WM, Orchardson R. Dentine hypersensitivity: Definition, prevalence, distribution and aetiology. Tooth Wear and Sensitivity, 2000: 239-248.

7. Addy M. Dentine hypersensitivity: A new perspectives on an old problem. Int Dent J, 2002; 52(5): 367-375.

8. Bartold PM. Dentinal hypersensitivity: A review. Aust Dent J, 2006; 51(3): 212-218.

9. Costacurta M, Docimo R, Montesani L, Maturo P. Comparing the efficacy in reducing dentin hypersensitivity of a new toothpaste containing $8.0 \%$ arginine, calcium carbonate, and $1.450 \mathrm{ppm}$ fluoride to a benchmark commercial desensitizing toothpaste containing $2 \%$ potassium ion: An eight-week clinical study in Rome, Italy. J Clin Dent, 2009; 20(4): 137-143.
10. Docimo R, Costacurta M, Montesani L, Maturo P. Comparing the efficacy in reducing dentin hypersensitivity of a new toothpaste containing $8.0 \%$ arginine, calcium carbonate, and $1.450 \mathrm{ppm}$ fluoride relative to a commercial sensitive toothpaste containing $2 \%$ potassium ion: An eightweek clinical study in Rome, Italy. J Clin Dent, 2009; 20(1): 17-22.

11. Holland GR, Narhi MN, Addy M, Gangarosa L. Guidelines for the design and conduct of clinical trials on dentine hypersensitivity. J Clin Periodontol, 1997; 24(11): 808-813.

12. Delgado E, Cummins D, Schiff T, Zhang YP. Clinical evaluation of the efficacy of an in-office desensitizing paste containing $8 \%$ arginine and calcium carbonate in providing instant and lasting relief of dentin hypersensitivity. Am J Dent, 2009; 22(A): 8A-15A.

13. Addy $M$, Dowell $P$, Dummer $P$. Dentine hypersensitivity: A etiology, differential diagnosis, and manegement. Br Dent J, 1985; 158(3): 92-96.

14. Orchardson R, Gillam DG. Managing dentin hypersensitivity. JADA, 2006; 137: 990-998. 